\title{
Deep and Comparative Transcriptome Analysis of Rice Plants Infested by the Beet Armyworm (Spodoptera exigua) and Water Weevil (Lissorhoptrus oryzophilus)
}

\author{
R. C. Venu • M. Sheshu Madhav • M. V. Sreerekha • Kan Nobuta • Yuan Zhang • \\ Peter Carswell • Michael J. Boehm • Blake C. Meyers • Kenneth L. Korth • \\ Guo-Liang Wang
}

Received: 14 December 2009/Accepted: 11 January 2010/Published online: 10 February 2010

(C) Springer Science+Business Media, LLC 2010

\begin{abstract}
The beet armyworm (Spodoptera exigua) and the rice water weevil (Lissorhoptrus oryzophilus) are two important insect pests in rice production. To identify insect-responsive genes in rice, we performed a deep transcriptome analysis of Nipponbare rice leaves infested with both beet armyworm and water weevil using massively parallel signature sequencing (MPSS). Many antisense, alternative, and novel transcripts were commonly and specifically induced and suppressed in the infested tissue. Key genes involved in the defense metabolic pathways such as salicylic acid and jasmonic acid biosynthesis pathways
\end{abstract}

R. C. Venu and M. Sheshu Madhav contributed equally to the project.

Electronic supplementary material The online version of this article (doi:10.1007/s12284-010-9037-8) contains supplementary material, which is available to authorized users.

R. C. Venu $\cdot$ M. Sheshu Madhav $\cdot$ M. V. Sreerekha

M. J. Boehm $\cdot$ G.-L. Wang $(\bowtie)$

Department of Plant Pathology, The Ohio State University,

Columbus, OH 43210, USA

e-mail: wang.620@osu.edu

K. Nobuta $\cdot$ B. C. Meyers

Delaware Biotechnology Institute, University of Delaware,

Newark, DE 19711, USA

Y. Zhang $\cdot$ P. Carswell

The Ohio Super Computer Center,

Columbus, $\mathrm{OH}$, USA

\section{K. L. Korth $(\bowtie)$}

Department of Plant Pathology, 217 Plant Science,

University of Arkansas,

Fayetteville, AR 72701, USA

e-mail: kkorth@uark.edu were up-regulated in the infested leaves. To validate the MPSS results, we analyzed the transcriptome of the rice leaves infested with water weevils using Solexa's sequencing-bysynthesis (SBS) method. The MPSS and SBS data were highly correlated (Pearson's correlation coefficient $=0.85$ ), and $83 \%$ of genes had similar gene expression in both libraries. Our comprehensive and in-depth survey of the insect-infested libraries provides a rich genomic resource for further analyzing the function of key regulatory genes involved in insect resistance in rice.

Keywords Beet armyworm · Water weevil · MPSS · SBS · Transcriptome analysis

\section{Introduction}

Herbivorous insects are responsible for destroying one fifth of the world's total annual crop production. Plants have evolved several layers of defense mechanisms against herbivorous insects (Mello and Silva-Filho 2002; Korth 2003). Understanding the molecular basis of these host mechanisms to insect attack is essential for effective control of insect damage in crop production. In the last decade, extensive research has revealed the expression pattern of defense-related genes in the infested plants by using different gene expression profiling technologies such as microarrays. Microarray-based genome-wide transcriptomic analyses have been performed in several plant species, including Arabidopsis thaliana (De Vos et al. 2005; Reymond et al. 2000, 2004; Stotz et al. 2000), bean (Arimura et al. 2000), Nicotiana attenuata (Voelckel et al. 
2004), Populus trichocarpa $\times$ Populus deltoides (hybrid poplar; Major and Constabel 2006; Ralph et al. 2006), Picea sitchensis (Sitka spruce; Ralph et al. 2006), Medicago truncatula (Leitner et al. 2005), and rice (Yuan et al. 2008). Although many commonly induced or suppressed defenserelated genes were identified in the plants infested with phloem-feeding or chewing insects in comparison with mechanical wounding, there were considerable differences in the transcriptomic response of infested plants to different insects (Zheng and Dicke 2008). For example, a similar number of differentially expressed genes $(\sim 200)$ were identified in Arabidopsis plants damaged by cell-content feeding thrips (Frankliniella occidentalis) and chewingbiting caterpillars (Pieris rapae), but the gene sets of those identified genes that responded to the two insects were quite different (De Vos et al. 2005). Interestingly, Arabidopsis plants showed a different defense response to insects with a similar feeding mode, such as aphids (Myzus persicae) and whiteflies (Bemisia tabaci) (Kempema et al. 2007). Moreover, transcriptomic changes in different cultivars after attack by the same insect were different (Broekgaarden et al. 2007). These results demonstrate the complexity of the defense mechanisms in plants after insect attack.

Plant defense responses to herbivory and wounding are often mediated by jasmonic acid (JA), salicylic acid (SA), and ethylene (ET; Walling 2000; Leon et al. 2001; Ryan 2000). For example, DNA microarray studies indicate that the JA pathway has a dominant role in regulating global changes in gene expression in response to both mechanical wounding and herbivory (De Vos et al. 2005; Devoto et al. 2005; Major and Constabel 2006; Ralph et al. 2006; Reymond et al. 2000, 2004). Emerging evidence indicates that phloem-feeding insects actively suppress jasmonatebased defenses (Thompson and Goggin 2006; Zarate et al. 2007). The role of SA in host resistance is less important with chewing insects than with phloem-feeding insects like aphids and brown plant hoppers, which induce SA-dependent responses (Zhang et al. 2004). ET also affects the expression of defensive proteins and secondary metabolites (Harfouche et al. 2006; Hudgins and Franceschi 2004; Winz and Baldwin. 2001). Relative to JA, however, ET production during herbivore attack is considered to play a minor role in the active defense response (von Dahl and Baldwin 2007). Transcript profiles elicited by phloem-feeding insects are markedly different from those induced by herbivorous insects from other feeding guilds and are generally associated with the activation of SA-responsive genes and weak expression of JA-responsive genes (De Vos et al. 2005; Gao et al. 2007; Kempema et al. 2007, Thompson and Goggin 2006).

In this study, we aimed to understand the common and specific transcriptional responses of rice plants to two important insect pests: the beet armyworm and the rice water weevil. The beet armyworm feeds by chewing, and the rice water weevil feeds by scraping. The beet armyworm occurs throughout the USA east of the Rocky Mountains and can be a sporadic pest on rice plants in the southeastern USA. The rice water weevil, which is a much more serious threat to rice production than the beet armyworm, is distributed throughout North and South America. In addition, this species is now found in many Asian countries, including Korea, Japan, and China, where it is considered one of the most important invasive insect pests on rice.

To profile the transcripts expressed in the rice plants $24 \mathrm{~h}$ after infestation by armyworm and water weevil, we used massively parallel signature sequencing (MPSS) and sequencing-by-synthesis (SBS) technologies. We identified many up- or down-regulated genes that were commonly or specifically expressed in the rice plants infested by armyworm and water weevil. Some of these genes belong to different metabolic pathways involved in the production of SA, JA, ET, and other secondary metabolites. Our results provide the first comprehensive view of the transcriptome changes after insect infestation in rice plants based on two high-throughput sequencing methods. The identified candidate genes are excellent starting materials for further elucidating the function of important genes involved in the rice and insect interactions.

\section{Results}

Library characteristics and sequence matching analysis

About 1.0 to 1.2 million individual 17-base signatures were obtained in the four MPSS libraries (PLA, PLW, PLC, and NLD, Table 1). These signatures were processed with reliability and significance filters as described by Meyers et al. (2004a). A total of 46,904 distinct 17-base signatures were obtained from the MPSS libraries (Table 1 and Fig. 1). To compare the expression levels across the libraries, we normalized the frequency of signatures in each library to one million (transcripts per million or TPM). Figure 1 shows the number of pooled distinct signatures from the four MPSS libraries separated by reliability and significance filters and further grouped based on their match in the Nipponbare genomic sequence. When all the unique, reliable, and significant signatures ( $\geq 4$ TPM) from the four libraries were clustered, a total of 37,532 unique signatures were obtained. Clustering of reliable significant signatures led to identification of 26,282 unique signatures that had only one hit in the genome (hits=1). A total of 5,358 reliable significant signatures matched the genome more than once (hits $>1$ ). About $5 \%$ of the signatures were significant unreliable, and $30 \%$ of them had genome 
Table 1 Statistics of Insect-Infested and Control Rice MPSS and SBS Libraries

\begin{tabular}{|c|c|c|c|c|c|}
\hline \multirow{2}{*}{$\begin{array}{l}\text { Technology } \\
\text { Signature } \\
\text { category }\end{array}$} & \multicolumn{4}{|l|}{ MPSS } & \multirow{2}{*}{$\begin{array}{l}\text { SBS } \\
\text { Water weevil-infeste } \\
\text { plants (SPLW) }\end{array}$} \\
\hline & $\begin{array}{l}\text { Beet armyworm- } \\
\text { infested plants (PLA) }\end{array}$ & $\begin{array}{l}\text { Water weevil-infested } \\
\text { plants (PLW) }\end{array}$ & $\begin{array}{l}\text { Mechanical wounded } \\
\text { plants (PLC) }\end{array}$ & $\begin{array}{l}\text { Unwounded control } \\
\text { plants (NLD) }\end{array}$ & \\
\hline Total sequenced & $1,150,869$ & $1,012,170$ & $1,213,577$ & $1,254,824$ & $3,051,005$ \\
\hline Distinct & 21,365 & 20,282 & 18,202 & 20,791 & 99,837 \\
\hline Reliable & 18,311 & 18,593 & 17,048 & 18,659 & 64,332 \\
\hline Unreliable & 3,054 & 1,689 & 1,154 & 2,132 & 35,505 \\
\hline Significant & 15,326 & 16,673 & 14,259 & 14,901 & 33,123 \\
\hline Nonsignificant & 6,039 & 3,609 & 3,943 & 5,890 & 66,714 \\
\hline Reliable significant & 14,480 & 15,912 & 13,779 & 14,395 & 31,955 \\
\hline Reliable nonsignificant & 3,831 & 2,681 & 3,269 & 4,264 & 32,377 \\
\hline Unreliable significant & 846 & 761 & 480 & 506 & 1,168 \\
\hline Unreliable nonsignificant & 2,208 & 928 & 674 & 1,626 & 34,337 \\
\hline Distinct genes expressed ${ }^{a}$ & 7,941 & 8,871 & 7,738 & 8,146 & 13,964 \\
\hline 1-100 ТРМ & 19,370 & 17,865 & 16,147 & 18,132 & 96,570 \\
\hline 101-1,000 ТРМ & 1,826 & 2,251 & 1,874 & 2,497 & 3,002 \\
\hline 1,001-10,000 ТРМ & 160 & 160 & 168 & 153 & 254 \\
\hline$>10,000$ ТРМ & 9 & 6 & 13 & 9 & 11 \\
\hline
\end{tabular}

${ }^{\mathrm{a}}$ Genome-matched reliable significant signatures

matches (Fig. 1). Distinct reliable significant signatures in PLA (14,480), PLW (15,912), PLC $(13,779)$, and NLD $(14,395)$ were identified. Thirteen transcripts with expression level $>10,000$ TPM were expressed in the PLC library (Table 1).

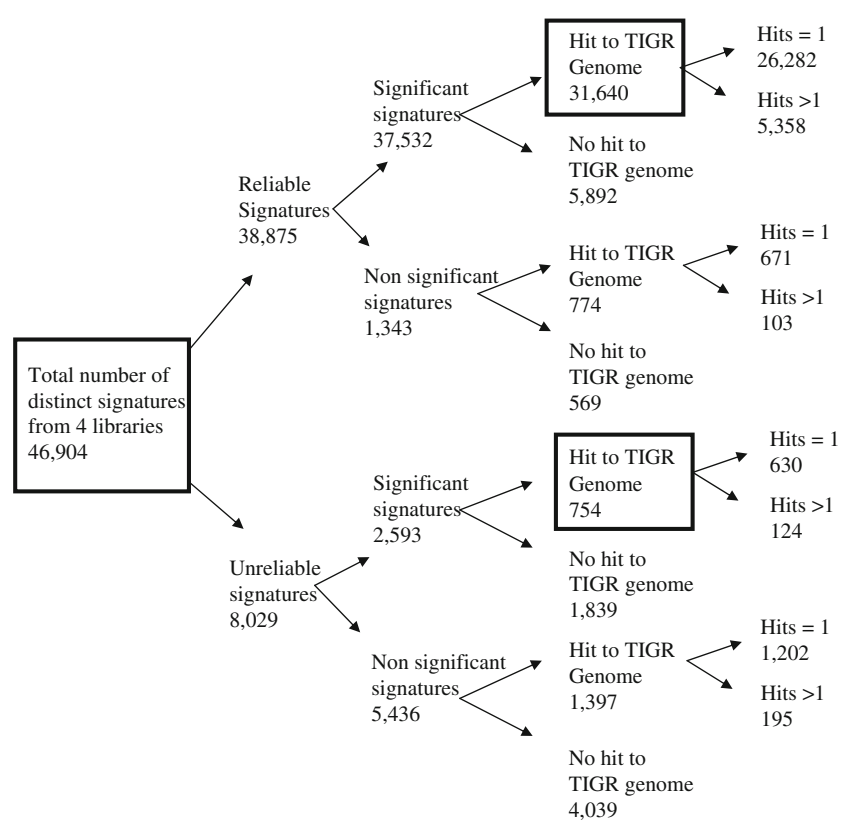

Fig. 1 Filter results for four MPSS libraries. A total of 46,904 distinct 17-base expressed signatures from four MPSS libraries were processed according to three filters-"significance," "reliability," and "genomic match"—as described by Meyers et al. (2004a).
All the reliable experimental signatures were matched to the rice genomic sequence to determine the precise location of expressed sense and antisense transcripts in the rice genome. About $87-90 \%$ of the signatures matched to the japonica (Nipponbare) genomic sequence (Table S2 of the Electronic Supplementary Material). Also, $80-83 \%$ of the signatures matched to rice annotated genes, of which nearly $73 \%$ and $15 \%$ signatures belonged to sense and antisense transcripts, respectively. Among them, about 5\% represented both sense and antisense signatures. About $86 \%$ of the signatures matched to the existing ESTs at the TIGR database. In addition, based on the precise location/matching of the experimental signatures on the annotated genes, the number of sense (classes 1, 2, 5, and 7) and antisense (classes 3 and 6) signatures were identified in PLA (11,182 and 2,025), PLW (11,858 and 2,008), PLC (10,779 and 1,925), NLD (8,935 and 1,432), and SPLW (13,813 and 3,378; Table 2).

Expression pattern of antisense, alternative, and novel transcripts, and transcripts of transcription factors (TFs) after insect infestation

About $63-68 \%$ of the reliable signatures in the two libraries generated from infested plants matched the KnowledgeBased Oryza Molecular Biological Encyclopedia (KOME) full-length (FL) cDNAs. Among the matched signatures, about $57-60 \%$ were sense signatures and $10 \%$ were antisense (Table 2, Table S2 of the Electronic Supplementary Material), and about 2\% matched both sense and antisense 
Table 2 Classification of the Reliable MPSS Signatures from PLA, PLW, and PLC Libraries Based on their Location on the Annotated Genes

\begin{tabular}{|c|c|c|c|c|c|c|c|c|c|c|}
\hline \multirow[t]{2}{*}{ Signature category ${ }^{\mathrm{b}}$} & \multicolumn{2}{|l|}{ PLA } & \multicolumn{2}{|l|}{ PLW } & \multicolumn{2}{|l|}{ PLC } & \multicolumn{2}{|l|}{ NLD } & \multicolumn{2}{|l|}{ SPLW } \\
\hline & $\begin{array}{l}\text { Total } \\
\text { signatures }\end{array}$ & $\begin{array}{l}\text { Grouped } \\
\text { by gene }^{\mathrm{a}}\end{array}$ & $\begin{array}{l}\text { Total } \\
\text { signatures }\end{array}$ & $\begin{array}{l}\text { Grouped } \\
\text { by gene }\end{array}$ & $\begin{array}{l}\text { Total } \\
\text { signatures }\end{array}$ & $\begin{array}{l}\text { Grouped } \\
\text { by gene }{ }^{a}\end{array}$ & $\begin{array}{l}\text { Total } \\
\text { signatures }\end{array}$ & $\begin{array}{l}\text { Grouped } \\
\text { by gene }\end{array}$ & $\begin{array}{l}\text { Total } \\
\text { signatures }\end{array}$ & $\begin{array}{l}\text { Grouped } \\
\text { by gene }\end{array}$ \\
\hline $\begin{array}{l}\text { Class } 1 \text { (exon, } \\
\text { sense strand) }\end{array}$ & 5,335 & 4,707 & 5,861 & 5,161 & 5,206 & 4,534 & 5,758 & 5,090 & 5,868 & 5,250 \\
\hline $\begin{array}{l}\text { Class } 2(500 \mathrm{bp} \\
\text { 3'-UTR) }\end{array}$ & 5,263 & 4,673 & 5,314 & 4,746 & 5,020 & 4,483 & 4,825 & 4,368 & 11,490 & 9,053 \\
\hline $\begin{array}{l}\text { Class } 3 \text { (exon, } \\
\text { antisense strand) }\end{array}$ & 1,862 & 1,635 & 1,836 & 1,628 & 1,771 & 1,559 & 1,471 & 1,336 & 3,634 & 3,044 \\
\hline $\begin{array}{l}\text { Class } 4 \text { (un-annotated } \\
\text { region) }\end{array}$ & 825 & 0 & 872 & 0 & 819 & 0 & 857 & 0 & 2,858 & 0 \\
\hline $\begin{array}{l}\text { Class } 5 \text { (intron, } \\
\text { sense strand) }\end{array}$ & 404 & 383 & 498 & 473 & 412 & 390 & 485 & 452 & 2,095 & 1,781 \\
\hline $\begin{array}{l}\text { Class } 6 \text { (Intron, } \\
\text { antisense strand) }\end{array}$ & 163 & 160 & 172 & 165 & 154 & 149 & 113 & 110 & 425 & 398 \\
\hline $\begin{array}{l}\text { Class } 7 \text { (span splice } \\
\text { site, sense strand) }\end{array}$ & 180 & 178 & 185 & 183 & 141 & 139 & 173 & 171 & 337 & 328 \\
\hline $\begin{array}{l}\text { Classes } 1,2,5,7 \\
\quad \text { (Sense signatures) }\end{array}$ & 11,182 & 8,786 & 11,858 & 9,284 & 10,779 & 8,407 & 11,241 & 8,935 & 19,790 & 13,813 \\
\hline $\begin{array}{l}\text { Classes } 3,6 \\
\quad \text { (antisense signatures) }\end{array}$ & 2,025 & 1,769 & 2,008 & 1,773 & 1,925 & 1,691 & 1,584 & 1,432 & 4,059 & 3,378 \\
\hline Total & 21,365 & 9,469 & 20,282 & 10,013 & 18,202 & 9,097 & 20,791 & 9,558 & 26,707 & 14,301 \\
\hline
\end{tabular}

${ }^{a}$ Grouped by gene including transposons

${ }^{\mathrm{b}}$ See Meyers et al. 2004a for class definitions

strands of the same full-length cDNA. Expression of the antisense transcripts was confirmed by matching the significant reliable signatures from each library with the rice antisense full-length cDNAs in the KOME database. The total number of genes with antisense transcripts was 1,769 in PLA, 1,773 in PLW, 1,691 in PLC, and 1,432 in NLD (Table 2), suggesting a significant induction of antisense gene expression in the insect-infested and wounded leaves. The specifically induced or suppressed genes with antisense in the PLA and PLW libraries were identified by comparison with the two control libraries (PLC and NLD). Forty antisense genes in PLA were $\geq 5$-fold induced, and none was suppressed relative to PLC and NLD, respectively (Table 3). Similarly, 44 and 3 genes were $\geq 5$-fold induced and suppressed in PLW, respectively, when compared to the two controls (Table 3). The identities of the genes encoding antisense transcripts with $\geq 5$-fold induction or suppression are listed in Table S3 of the Electronic Supplementary Material.

Table 3 Specifically Induced or Suppressed (Fivefold or More) Antisense Genes (with KOME Antisense Transcripts Support), Alternative Transcripts (with TIGR Alternative Transcripts Support)
About $10-12 \%$ of the expressed genes showed alternative splicing when compared with the TIGR alternative-spliceform clusters. Among them, the genes with alternative transcripts that were induced or suppressed specifically in PLA and PLW relative to the two controls were identified (Table 3). A total of 223 and 40 specifically induced genes ( $\geq 5$-fold induction) produced alternative transcripts in PLA and PLW, respectively. Some of the pathogen defense-related genes, such as those encoding metallothionein-like protein type 2 (TC334871, TC323823, TC320546, TC311902, TC319184), nonspecific lipid transfer protein (TC327106), aspartic proteinase precursor (TC300646), BTH-induced protein phosphatase 1 (TC300268), thioredoxin (TC304211), calmodulin (TC338165), catalase (TC342436), Rho-GTPaseactivating protein (TC355505), cysteine proteinase inhibitor 2 (TC315144), small GTP-binding protein (TC335268), and stress-related protein (TC341761), generated alternative transcripts (Table S3 of the Electronic Supplementary Material).

and Genes Encoding Transcription Factors in Insect-Infested Plants Compared to Wound and Unwound Plants

\begin{tabular}{|c|c|c|c|c|c|c|}
\hline \multirow[t]{2}{*}{ Library } & \multicolumn{2}{|c|}{ Antisense genes } & \multicolumn{2}{|c|}{ Alternative transcripts } & \multicolumn{2}{|c|}{ Transcription factor genes } \\
\hline & Induced & Suppressed & Induced & Suppressed & Induced & Suppressed \\
\hline PLA & 40 & 0 & 223 & 15 & 137 & 94 \\
\hline PLW & 44 & 3 & 40 & 71 & 166 & 80 \\
\hline
\end{tabular}


The novel transcripts that matched the genome sequence but were not present in the KOME FL-cDNAs and TIGREST databases, and the novel genes that matched the genome sequence but were not present in the TIGR ESTs, KOME FL-cDNAs, and TIGR annotated rice genes, were searched in both PLA and PLW. About 1,000 novel transcripts and 1,200-1,300 novel genes were identified (Table S3 of the Electronic Supplementary Material).

The TF genes that were induced or suppressed in PLA or PLW compared to PLC and NLD are given in the Table S4 of the Electronic Supplementary Material. Some of the important stress-related transcription factor genes encoding LIM domain-containing protein (Os06g13030), heat shock protein (Os03g63750), zinc finger domain protein (Os03g55540), homeobox-leucine zipper protein (Os10g39030), and Mybrelated transcription factor (Os01g09280) were highly induced in both PLA and PLW relative to PLC and NLD. However, some of the NAC domain-containing transcription factor genes (Os11g08210 and Os02g36880) were suppressed in both PLA and PLW libraries compared to PLC and NLD.

Promoter analysis of the genes responsive to insect infestation

Promoter analysis revealed the presence of many conserved cis motifs in the upstream regions of the up-regulated genes. In the 12 highly induced genes ( $\geq 50$-fold) in both PLA or PLW, 17 types of cis motifs were identified (Table 4 and Table S4 of the Electronic Supplementary Material). These motifs were highly represented in the promoters of the plus or minus strand of the 12 defense-related genes. The precise locations of the known cis elements in the promoter regions of all 12 genes are listed in the Table S4 of the Electronic Supplementary Material.

Identification of genes in the defense-related metabolic pathways

A network map of defense-related metabolic pathways was generated based on the biochemical pathways reported at the Gramene website (http://www.gramene.org/; Fig. 2). The important metabolic pathways responsible for the production of secondary metabolites including SA, JA, ET, and other hormones were integrated based on the genes identified in the four MPSS libraries. Genes that were at least 5-fold upor down-regulated in PLA and PLW (relative to PLC and NLD) and that were involved in the production of these defense molecules are presented. Many genes involved in the biosynthesis of JA, like lipoxygenases (Os12g37290, Os08g39850, Os04g37430) and 12-oxophytodienolate reductase (Os06g11240), were up-regulated in both PLA and PLW libraries (Fig. 2). The key gene encoding phenylalanine ammonia-lyase (Os04g43760), which catalyzes the biosyn- thesis of SA through L-phenylalanine, was up-regulated in both PLA (36-fold) and PLW (44-fold). In contrast, the gene encoding isochorismate synthase 1 (Os09g19734), which produces SA through chorismate, was down-regulated in both PLA (81-fold) and PLW (72-fold). However, many of the genes belonging to ET biosynthesis were downregulated in both PLA and PLW, such as those encoding 1-aminocyclopropane-1-carboxylate synthase (Os01g55540, 58-fold), centromere/kinetochore protein zw10 (Os11g34310, 5-fold), tyrosine aminotransferase (Os11g42510, 14-fold), tyrosine transamines (Os10g25140, 14-fold, Os09g28050, 6-fold), and 1-aminocyclopropane-1-carboxylate oxidase (Os09g27820, 25-fold). A large group of genes involved in brassinosteroid production, cytokinin production 7- $\mathrm{N}$ glucoside biosynthesis, and phenylpropanoid biosynthesis were also highly expressed.

Genes commonly expressed in both beet armyworm- and water weevil-infested plants but not in wounded or untreated control plants

A total of 878 transcripts (653 genes) were 5-fold or more up-regulated and 371 transcripts (340 genes) were 5-fold or more down-regulated in both PLA and PLW, relative to those in the two control libraries (Fig. 3; Table S5 of the Electronic Supplementary Material). Among them, the known defense genes with 5-fold induction and commonly or specifically present in the two libraries from insect-infested rice are listed in Table 5. Among the defense genes, we observed the up-regulation of the genes encoding BowmanBirk protease inhibitors (Os01g60730, Os01g04050), lipoxygenase (Os12g37260), nucleic acid binding protein (Os03g07370), terpene synthase 8 (Os04g27790), OsWRKY78 - superfamily of rice TFs having WRKY and zinc finger domains (Os07g39480), metallothionein-like protein type 2 (Os01g05650), RING-H2 finger protein (Os01g60730), cysteine-rich receptor-like protein kinase (Os07g43560), and 4-coumarate-CoA ligase (Os01g67530; Table 5). Other genes belonging to secondary metabolite production were also up-regulated, including those encoding squalene monooxygenase (Os03g12910), tyrosine decarboxylase gene (Os07g25590), phenylalanine ammonialyase gene (Os04g4376), and $N$-acylethanolamine amido hydrolase (Os11g06900; Table 5; Table S5 of the Electronic Supplementary Material). Some of the genes involved in the protein degradation pathway were up-regulated in the plants infested with either pest but not in the wounded and untreated plants (Table 5; Table S3 of the Electronic Supplementary Material); these genes included $26 \mathrm{~S}$ protease regulatory subunit 7 (Os06g09290), brix domaincontaining proteins (Os01g33030), hexose carrier protein HEX6 (Os10g41190), tab2 protein (Os02g39740), F-box domain-containing proteins (Os09g32870, Os08g09760, 


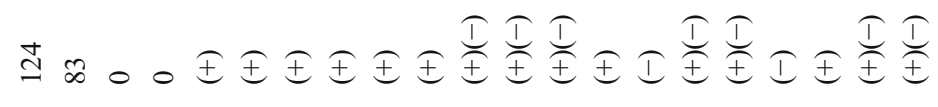

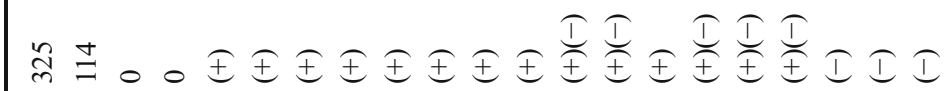

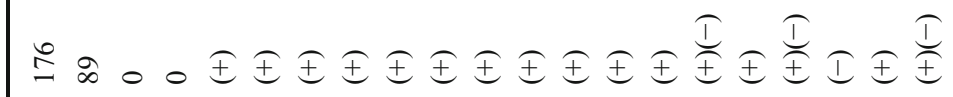

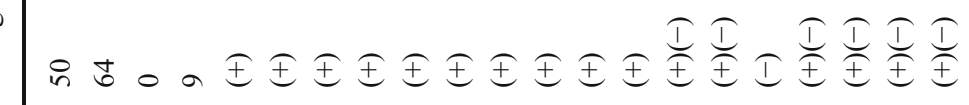
(1)

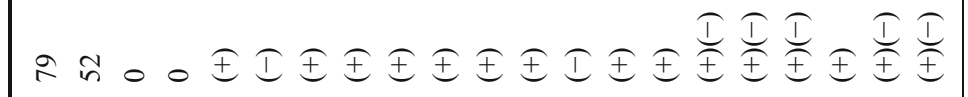

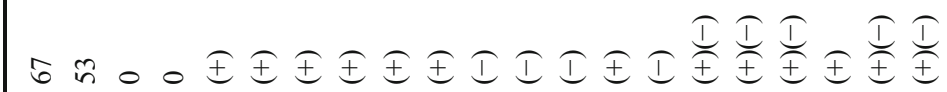
(1)

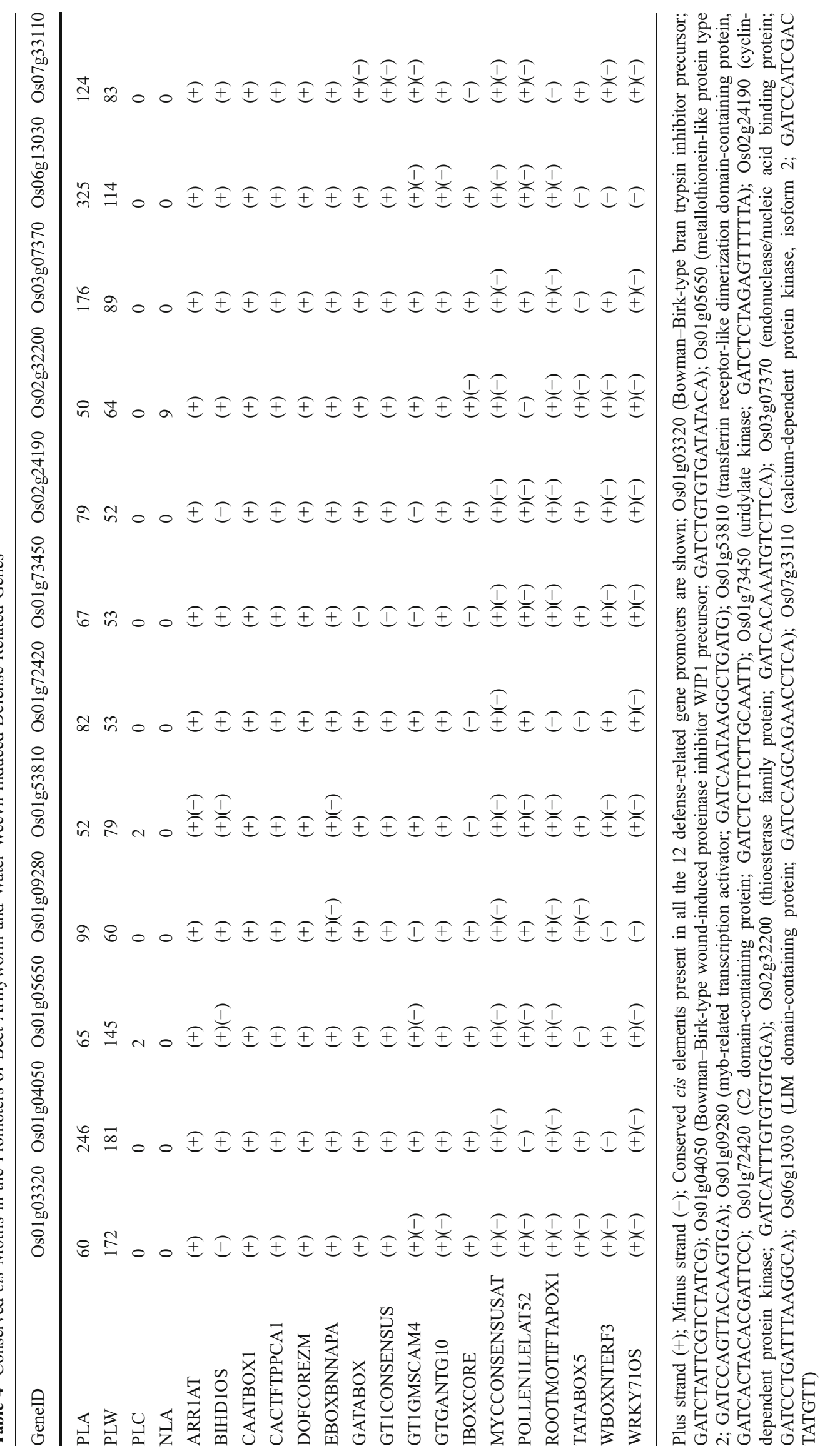




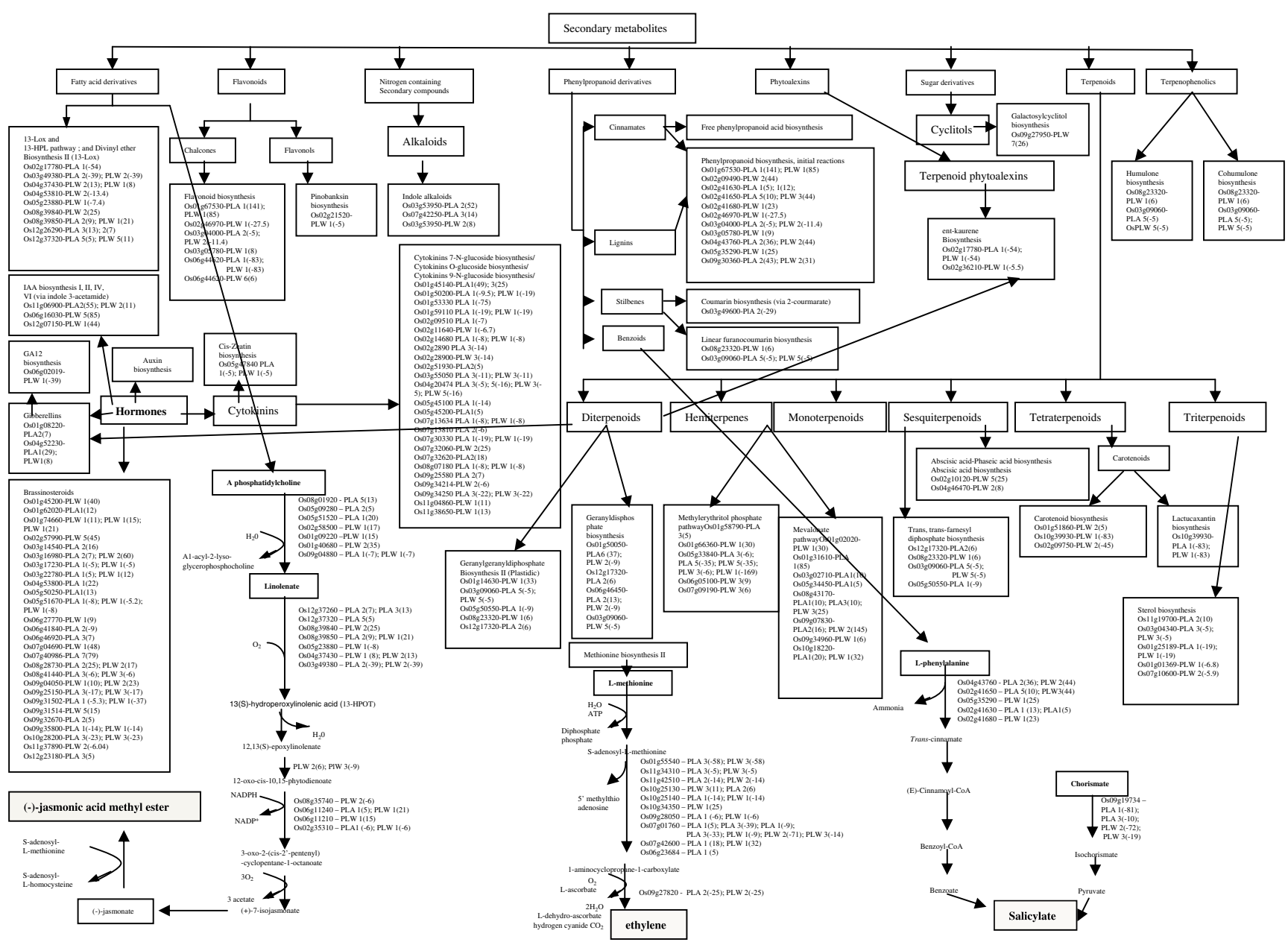

Fig. 2 Network of defense-related pathways showing the expression or suppression of key genes belonging to metabolism of secondary metabolites including salicylic acid, jasmonic acid, ethylene, and hormones. The genes that were up- or down-regulated 5-fold in PLA or PLW are shown in parenthesis (positive numbers indicate upregulated genes and negative numbers indicate down-regulated genes). The number next to the library code (PLA or PLW) shows the signature class as described by Meyers et al. (2004a).

metabolism of cofactors and vitamins, carbohydrate metabolism, and energy metabolism were up-regulated in both kinds of insect-infested plants (Fig. S1 and Table S5 of the Electronic Supplementary Material).

We also observed the induction of the $\mathrm{NAD}(\mathrm{P}) \mathrm{H}-$ dependent oxidoreductase gene (Os04g08550 and its six isoforms), which encodes a key enzyme involved in radical scavenging and the accumulation of reactive oxygen species. The induction seems to be specific to both insect infestations because expression of these genes did not increase in the mechanically damaged plants. The transcripts encoding several key JA biosynthetic enzymes like allene oxide synthase, allene oxide cyclase, and phospholipase D were up-regulated in rice after infestation by either insect (Fig. 2; Table 5; Table S5 of the Electronic Supplementary Material). Up-regulation was identified for several isoforms of the phenylalanine ammonia-lyase genes (Os04g43760, Os02g41650, Os05g35290, Os02g41630, and Os02g41680) involved in the SA biosynthesis pathway 
Table 5 List of Defense-Related Genes Specifically and Commonly Induced in the Host After Beet Armyworm and Water Weevil Infestations

\begin{tabular}{|c|c|c|c|c|c|c|c|}
\hline ID & Signature & PLA & PLW & PLC & NLD & Gene ID & Gene description \\
\hline \multicolumn{8}{|c|}{ Genes commonly induced in both PLA and PLW } \\
\hline 1 & GATCTGTGTGATATACA & 246 & 181 & 0 & 0 & Os01g04050 & $\begin{array}{l}\text { Bowman-Birk-type wound-induced proteinase inhibitor } \\
\text { WIP1 precursor }\end{array}$ \\
\hline 2 & GATCGATTTCATTTGGG & 206 & 119 & 11 & 0 & Os05g31750 & Annexin-like protein RJ4 \\
\hline 3 & GATCACAGTGTAGCGTG & 178 & 526 & 2 & 0 & Os12g37260 & Lipoxygenase 2.1, chloroplast precursor \\
\hline 4 & GATCCTGATTTAAGGCA & 176 & 89 & 0 & 8 & Os03g07370 & Endonuclease/nucleic acid binding protein \\
\hline 5 & GATCTGTAATTCGAGTT & 146 & 218 & 0 & 0 & Os07g43560 & CRK10 \\
\hline 6 & GATCGTCGCGGAGGTGG & 101 & 130 & 0 & 0 & Os02g50770 & Peroxidase 65 precursor \\
\hline 7 & GATCGTGTGGTGGAGAG & 82 & 132 & 2 & 0 & Os04g27790 & Terpene synthase 8 \\
\hline 8 & GATCATCAGAATTTGGT & 70 & 102 & 18 & 3 & Os07g39480 & $\begin{array}{l}\text { OsWRKY78 - superfamily of rice TFs having WRKY } \\
\text { and zinc finger domains }\end{array}$ \\
\hline 9 & GATCCTGCCACTTGCCC & 69 & 127 & 0 & 17 & Os08g09860 & FMN-dependent dehydrogenase family protein \\
\hline 10 & GATCCAGTTACAAGTGA & 65 & 145 & 2 & 0 & Os01g05650 & Metallothionein-like protein type 2 \\
\hline 11 & GATCATCCTCGCGGCGC & 60 & 141 & 4 & 0 & Os01g60730 & RING-H2 finger protein ATL5A \\
\hline 12 & GATCTATTCGTCTATCG & 60 & 172 & 0 & 0 & Os01g03320 & Bowman-Birk-type bran trypsin inhibitor precursor \\
\hline \multicolumn{8}{|c|}{ Genes specifically induced in PLA } \\
\hline 13 & GATCATGTAAACTGTGG & 231 & 0 & 0 & 0 & Os07g40860 & Vegetative cell wall protein gp1 precursor \\
\hline 14 & GATCCATGGGCTGTACT & 206 & 0 & 2 & 0 & Os08g44020 & Lyase \\
\hline 15 & GATCAGTGGCAAGAAAC & 174 & 0 & 0 & 0 & Os12g44310 & $9,10-9,10$ carotenoid cleavage dioxygenase 1 \\
\hline 16 & GATCTCTGCGCATGGTT & 170 & 0 & 0 & 0 & Os03g22810 & Superoxide dismutase 1 \\
\hline 17 & GATCGACTTCTCCCATC & 124 & 0 & 0 & 0 & Os06g24990 & Xylanase inhibitor protein 1 precursor \\
\hline 18 & GATCGGCCACGACGACA & 111 & 0 & 0 & 0 & Os07g01660 & Disease resistance response protein 206 \\
\hline 19 & GATCCGATGCTGTGTTG & 103 & 25 & 12 & 0 & Os08g04170 & Zinc finger $\mathrm{C}-\mathrm{x} 8-\mathrm{C}-\mathrm{x} 5-\mathrm{C}-\mathrm{x} 3-\mathrm{H}$ type family protein \\
\hline 20 & GATCAACGAATTCAGCC & 146 & 44 & 4 & 0 & Os02g41860 & Aquaporin PIP2.2 \\
\hline \multicolumn{8}{|c|}{ Genes specifically induced in PLW } \\
\hline 21 & GATCTGCGATGAACTGA & 0 & 212 & 0 & 3 & Os05g11320 & Metallothionein-like protein type 3 \\
\hline 22 & GATCCACACAGTATAGC & 0 & 195 & 0 & 0 & Os06g16420 & Amino acid transporter-like protein \\
\hline 23 & GATCTCAGGGCGGAGGC & 0 & 160 & 0 & 0 & Os02g53420 & Heat shock $70 \mathrm{kDa}$ protein, mitochondrial precursor \\
\hline 24 & GATCGAGCGCGCGTTCG & 0 & 249 & 0 & 0 & Os07g07320 & Glutathione-S-transferase GSTU6 \\
\hline 25 & GATCAGCAGGATTAGGT & 0 & 140 & 6 & 5 & Os02g42690 & Zinc finger, C3HC4-type family protein \\
\hline 26 & GATCCTATGTTCAAAGA & 9 & 147 & 0 & 0 & Os02g40240 & Leucine-rich repeat receptor protein kinase EXS precursor \\
\hline 27 & GATCGCTCAATTTTTCC & 9 & 141 & 11 & 13 & Os05g48970 & C-terminal zinc finger \\
\hline 28 & GATCATCTCGGCCGGGT & 9 & 251 & 7 & 13 & Os04g57880 & DnaJ domain-containing protein \\
\hline 29 & GATCTGTTTTGTTTGGT & 2 & 108 & 14 & 10 & Os06g03800 & Ankyrin repeat domain-containing protein 28 \\
\hline 30 & GATCCCCAAGTCGGCGT & 11 & 110 & 4 & 0 & Os02g46970 & 4-coumarate-CoA ligase 2 \\
\hline
\end{tabular}

(Fig. 2; Table S5 of the Electronic Supplementary Material). In addition, calmodulin (Os06g06160) and a calciumbinding protein were also induced in plants infested with either insect (Table S5 of the Electronic Supplementary Material).

Genes differentially expressed in beet armyworm- and water weevil-infested plants

A total of 1,666 transcripts (1,570 genes) were specifically upregulated (i.e., up-regulated in one kind of insect-infested plant but not the other), and 587 transcripts (580 genes) were specifically down-regulated in PLA compared with the two controls (Fig. 3 and Table S5 of the Electronic Supplementary Material). Similarly 2,033 transcripts (1,863) were specifically up-regulated, and 444 transcripts (432 genes) were specifically down-regulated in the PLW library (Fig. 3 and Table S5 of the Electronic Supplementary Material). The genes encoding transcription factors containing known domains such as MADS, PLATZ, RWP-RK, SET, and ZIM were highly up-regulated in the beet armyworm-infested plants (Table 5 and Tables S4 and S5 of the Electronic Supplementary Material), whereas the genes encoding transcription factors with ABI3VP1, ARF, ARID, AUX/ IAA, SNF2, SBP, TCP, TUB, and WRKY domains were highly up-regulated in water weevil-infested plants (Table 5 
and Tables S4 and S5 of the Electronic Supplementary Material). Defense- or metabolism-related genes encoding vegetative cell wall protein gp1 (Os07g40860), 9,10 carotenoid cleavage dioxygenase (Os12g44310), superoxide dismutase (Os03g22810), fungal xylanase inhibitor (Os06g24990), and aquaporin PIP2.2 (Os02g41860) were specifically upregulated in the beet armyworm-infested plants (Table 5 and Table S5 of the Electronic Supplementary Material). Upregulation of some defense- or metabolism-related genes also occurred in the water weevil-infested plants; these genes encoded metallothionein-like protein type 3 (Os05g11320), glutathione-S-transferase (Os07g07320), zinc finger, C3HC4-type family protein (Os02g42690), leucine-rich repeat receptor protein kinase (Os02g40240), ankyrin repeat domain-containing protein (Os06g3800), and 4-coumarateCoA ligase 2 (Os02g46970; Table 5 and Table S5 of the Electronic Supplementary Material).

\section{Validation using RT-PCR and SBS}

The expression pattern of 14 genes randomly selected from the PLA and PLW libraries were further evaluated using RTPCR (see the gene list in Table S1 of the Electronic Supplementary Material). Four up-regulated and three down-regulated genes in PLA or PLW compared to the two controls were analyzed through RT-PCR. About $65 \%$ of the genes (nine genes) showed a similar expression pattern in both RT-PCR and MPSS data (Fig. 4a). It is noteworthy that the gene encoding allene oxide synthase (a marker gene for JA synthesis) and the gene encoding phenylalanine ammonia-lyase (a maker gene for SA synthesis) showed up-regulation in plants infested with both insects compared to the controls (Fig. 4a).

The SBS library SPLW was constructed using the same RNA used to construct the PLW MPSS library. A total of

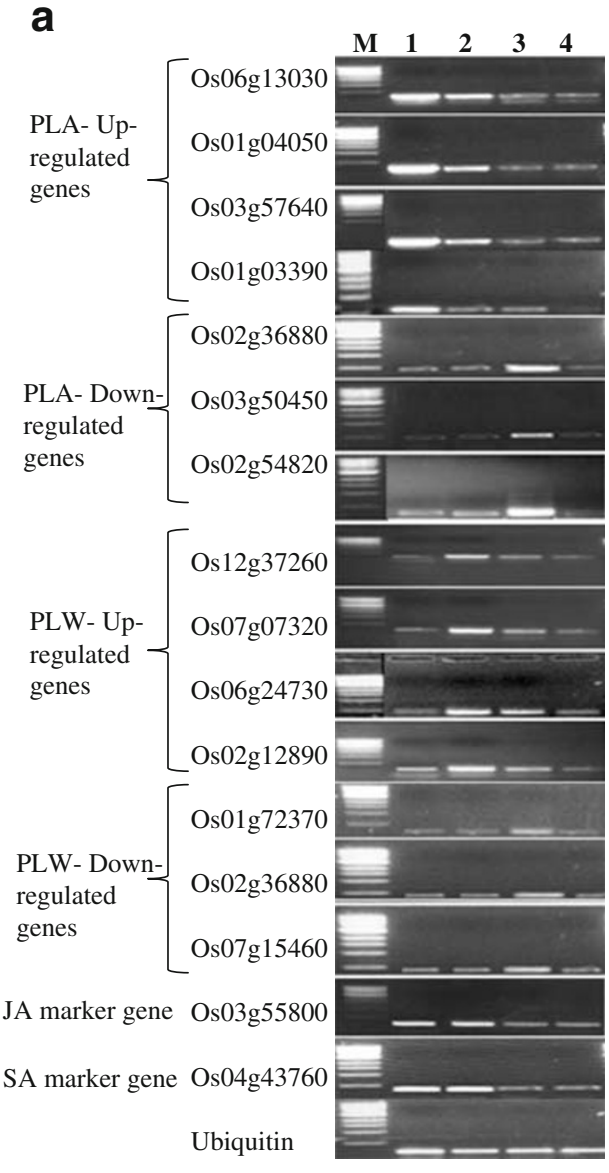

Fig. 4 Validation of MPSS data using RT-PCR and SBS analyses. a Validation of the MPSS tags identified in PLA and PLW using RTPCR analysis. The RNA isolated from leaves of plants that were infested with beet armyworm (1), infested with water weevil (2), mechanically wounded (3), or unwounded (4) was used. $M$ 1-kb size ladder. Ubiquitin gene was amplified as a loading control. b Commonly and specifically expressed genes in PLW (MPSS) and
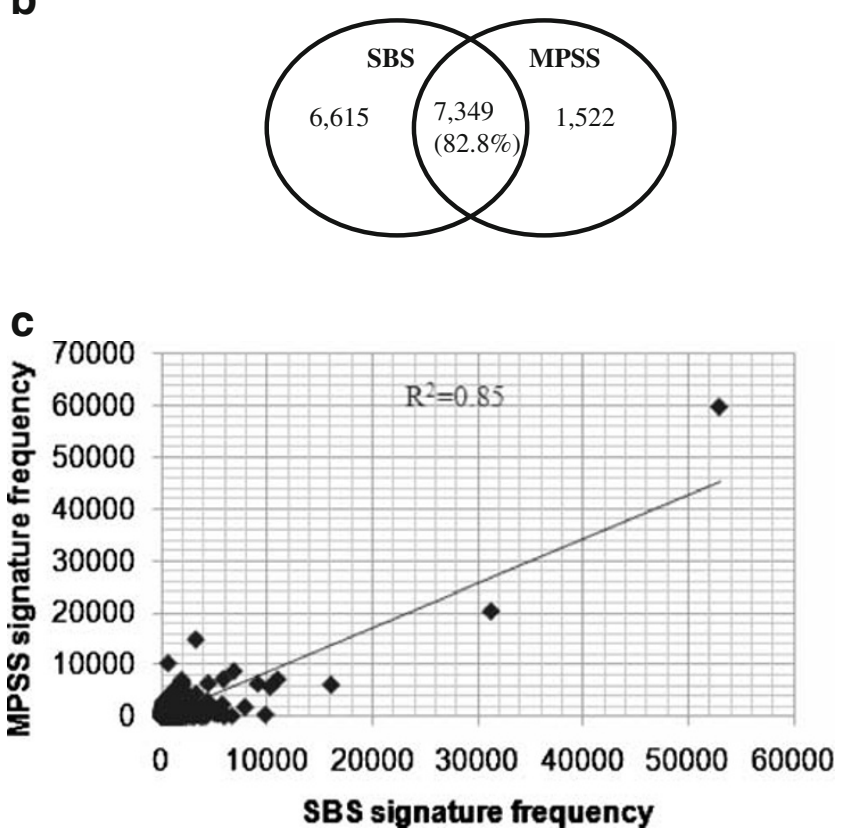

SPLW $(S B S)$. The distinct expressed genes identified in the SBS and MPSS libraries were used in the analysis. c Correlation of the gene expression patterns between the MPSS and SBS libraries. The genome-matched reliable and significant signatures from both MPSS and SBS libraries were subjected to Pearson's correlation analysis. Sixteen outliers that affected the correlation were removed based on regression analysis as described in Gowda et al. (2006). 
three million signatures were obtained from the library, which is 3 -fold greater than the number of reads in the PLW library (Table 1). The number of reliable significant signatures was about 2-fold greater in SPLW than in PLW $(31,995$ vs 15,912$)$. Many of the low-copy signatures (1100 TPM) were identified in the SBS library $(96,570$ in SPLW vs 17,865 in PLW). When the number of the annotated genes with reliable significant signatures was compared, the SBS library had about $57.4 \%$ more genes $(13,964)$ than the MPSS library $(8,871$; Fig. 4 b, Table 1$)$, suggesting a much deeper coverage in the SBS library for transcriptome survey. Between the matched annotated genes in the two libraries, $7,349(83 \%)$ genes were present in both libraries. The reliable significant signatures from SPLW and PLW libraries were compared using Pearson's correlation coefficient. A moderate correlation coefficient (0.65) was observed when MPSS and SBS expression data were compared without removal of any outlier transcripts. After removal of four outlier transcripts, the correlation coefficient was high $(0.85$; Fig. 4c; Table S6 of the Electronic Supplementary Material).

\section{Discussion}

With expected changes in climate and rice cropping systems, insect pests on rice are likely to become more epidemic and destructive in the future. Although insecticides are effective, undesirable environmental effects of insecticides and insect resistance to insecticides are becoming serious concerns in rice growing regions. It is clear that development of highly resistant cultivars is essential for sustainable rice production. However, the molecular basis of host resistance to insects in rice remains largely unknown. Yuan et al. (2008) identified 196 rice genes whose expression was significantly up-regulated by fall armyworm (Spodoptera frugiperda) caterpillars using a half-genome rice oligo microarray. The current study used two high-throughput sequencing techniques to provide the first large-scale and deep transcriptome analysis of rice plants infested with two insect pests. The deep-sequencing capacity of both techniques assured the collection of most transcripts in the rice tissues. Although MPSS and SBS are two different platforms, the transcriptomes generated by the two methods were highly correlated in our study. Many genes commonly or specifically induced or suppressed in the plants infested by the two insects have been identified. Novel genes were also obtained with antisense and alternative transcripts that are specifically expressed in the infested tissues. In addition, many highly and specifically expressed $\mathrm{TF}$ genes were found in the infested rice plants, and these genes may play important roles in regulating or coordinating insect-defense pathways or networks in rice.
Further elucidation of the function of these genes in host defense against insects will provide new insights into the molecular basis of insect resistance and novel genes for engineering insect-resistant rice.

We found that many genes involved in host-defense signaling pathways generate antisense transcripts after insect infestation. This kind of phenomenon was also observed in rice infected with the fungal pathogen Magnaporthe oryza (Gowda et al. 2007). However, the function of antisense genes in plant defense against pathogens and insects is unclear. In addition, we also observed alternative splicing in about $18 \%$ of the rice genes in the libraries of insect-infested rice and in about $14 \%$ of the rice genes in the library of uninfested rice. The importance of alternative splicing in the resistance to pathogens was found in tobacco and Arabidopsis; when the derivative of alternative splicing of the tobacco $N$ gene and the Arabidopsis RPS4 genes was silenced, the level of the $N$ - and RPS4-mediated resistance was reduced or abolished (Jordan et al. 2002). In addition, $\mathrm{R}$ gene alternative splicing was dynamic during the defense response (Gassmann 2008). The function of both antisense and alternative transcripts identified in this study requires further detailed analysis in the defense response of plants to insect attack.

Terpenes are an important class of defense compounds that accumulate in plants after pathogen infection or arthropod-induced injury. Previous research has shown that Lepidopteran herbivory and oral factors induced transcripts encoding novel terpene synthases in $M$. truncatula (Gomez et al. 2005; Bede et al. 2006). Recently, Yuan et al. (2008) confirmed the induction of expression of seven of the 11 terpene synthase genes after fall armyworm infestation that was identified through the microarray experiments. Enzymes encoded by three TPS genes, Os02g02930, Os08g07100, and Os08g04500, were also biochemically characterized. In the current study, terpene synthase genes were induced in the host after both beet armyworm and water weevil infestations. In addition, we observed the induction of the Bowman-Birk family of proteinase inhibitors (BBPI) in both PLA and PLW libraries. BBPIs might contribute to plant defense against insect attack by inhibiting digestive enzymes of various insects. Transgenic plants expressing a BBPI gene had enhanced resistance to herbivory (Hilder et al. 1987). Genetic manipulation of the BBPI genes in transgenic rice may lead to new methods for insect control in rice production.

Various transcriptome analyses indicated that insect feeding elicits defense response in the host through SA-, JA-, and ET-regulated genes (Walling 2000; Moran et al. 2002; de Vos et al. 2007). The feeding of brown plant hoppers on rice up-regulates several genes involved in phenylpropanoid biosynthesis and genes required for sesquiterpene synthesis (Zhang et al. 2004; Cho et al. 
2005). In tomato, aphid infestation up-regulates SA signaling ( $\mathrm{Li}$ et al. 2006) while in Arabidopsis SA has been shown to have a neutral and negative effect on aphid and silver leaf whitefly growth, respectively (Pegadaraju et al. 2005; Zarate et al. 2007). Chewing insects largely induce JA because of the extensive damage caused by chewing (Howe 2004; Kessler and Baldwin 2002; Halitschke et al. 2003). In our study, many JA and SA biosynthetic genes were up-regulated in both PLA and PLW libraries, including the genes encoding phosphatidylcholine and linolenate 13(S)-hydroperoxylinolenic acid in the JA pathway and the genes encoding phenylalanine ammonia-lyase gene in the SA pathway. This up-regulation suggests an important role of both JA and SA in the response of rice to insect infestation. Endogenous ET has been shown to act as a cross-talk regulator with JA (Penninckx et al. 1998; Leon et al. 2001; Arimura et al. 2005, 2008). Enhanced production of ET has been reported in aphid-infested barley, which indicates active biosynthesis of this phytohormone in response to minimal wounding (Argandona et al. 2001). In the current study, however, the role of the ET-mediated signaling in insect-infested rice plants was unclear because the expression of the ACC synthase and ACC oxidase genes in the ET pathway was down-regulated. Nevertheless, the role of SA, JA, ET, and their cross-talks in the rice insect defense warrants further in-depth investigation.

MPSS has been used for whole genome transcription analysis in the last decade and has generated abundant data concerning expression in many organisms (Vega-Sanchez et al. 2007; Simon et al. 2009). Its complicated library construction procedure and high sequencing cost are two main limiting factors for the use in individual laboratories. As the cost of the next-generation sequencing methods has significantly decreased in the last few years, SBS sequencing has become a popular method for transcriptome analysis. To validate our MPSS results, we made and sequenced an SBS library using the same RNA sample that was used for the PLW library. Comparison analysis showed that about $83 \%$ of the genes were expressed in both MPSS and SBS libraries. Pearson's correlation analysis showed a high level of similarity (coefficient $=0.85$ ) in expression patterns of genes between these two platforms. However, SBS is a much better choice for transcriptome analysis because it costs $90 \%$ less than MPSS and generates 3-fold more transcripts. Furthermore, about $30 \%$ more transcripts have been found in the SBS library than in the MPSS library. Many of these additional signatures are low-copy transcripts, indicating that SBS is a powerful method for identifying race transcripts. As the sequencing cost for SBS is further reduced in the future, SBS will likely become a routine transcriptomic analysis for many biological experiments.

\section{Methods}

Insect rearing, plant growth conditions, and insect infestations

Beet armyworm larvae were reared in the laboratory, and neonates were maintained on rice plants before third-instar larvae were used in the experiment. Rice water weevils were collected as adults from the field and maintained on rice plants in the greenhouse. Nipponbare rice plants (Oryza sativa) were grown in a greenhouse. When the plants were 6 weeks old, they were individually placed in 24 cages. Insects (100 army worms or 500 weevils per cage) were added to 12 of the cages (six cages for each kind of insect). When the insects were added to cages, the plants in six other cages were mechanically damaged with a hole punch; 2-5 $\mathrm{mm}$ were removed from leaf edges, and care was taken to avoid damaging the mid-vein. Leaves were damaged at intervals of approximately $4 \mathrm{~cm}$ along the leaf edge, and the treatment was repeated $30 \mathrm{~min}$ after the initial damage. The plants in the six remaining cages were untreated controls, i.e., they did not experience insect infestation or mechanical damage. All leaf tissue from all 24 cages was collected $24 \mathrm{~h}$ after the insects had been added to the cages and after the leaves had been initially wounded. Conditions during this 24-h period were the same as described earlier in this section.

\section{RNA isolation and RT-PCR}

Total RNA was isolated using TRIzol reagent according to the manufacturer's instructions. RT-PCR was performed as reported previously (Venu et al. 2007). PLA, PLW, PLC, and NLD refer to the libraries of plants infested with the beet armyworm, plants infested with the water weevil, mechanically wounded plants, and unwounded control plants, respectively (Table 1). Selected candidate genes that were up- or down-regulated in these four libraries were amplified by gene-specific primers, which are listed in Table S1 of the Electronic Supplementary Material.

\section{MPSS and SBS library construction and bioinformatics}

The total RNA isolated from beet armyworm-infested plants, water weevil-infested plants, mechanically wounded plants, and untreated control plants was used for the construction of MPSS libraries. In addition, the same RNA for the PLW library was used for the construction of the SBS (SPLW) library. The MPSS libraries were constructed and sequenced essentially as previously described (Brenner et al. 2000; Meyers et al. 2004a; b; Nobuta et al. 2007). The SBS library was constructed according to manufacturer's (Illumina) instructions with minor modifications. All data from the 
MPSS and SBS libraries are deposited at our public websites: http://mpss.udel.edu/rice/ and http://mpss.udel. edu/rice_sbs. The study used rice reference sequence (RefSeq) databases such as TIGR ESTs release version 17.0 (http://compbio.dfci.harvard.edu/tgi/cgi-bin/tgi/gimain. pl?gudb=rice), KOME FL-cDNA sequences $(14$, http:// cdna01.dna.affrc.go.jp/cDNA), and release 5 of the TIGR pseudomolecules (23 January 2007) (ftp://ftp.tigr.org/pub/ data/Eukaryotic_Projects/o_sativa/annotation_dbs/pseudo molecules/version_5.0). The potential or "virtual" signatures were derived from the rice genome by extracting all occurrences of GATC plus the 14 nt sequence at the $3^{\prime}$ terminus (16 nt in case of SBS). These signatures were used for matching analysis with the experimental MPSS or SBS signatures obtained in this study. All the virtual genomic signatures derived from the rice genome were assigned a "class" based on the position of the signature relative to annotated genes (Meyers et al. 2004a). Signatures that did not match to the genome corresponded to the "Class 0" signatures and those that matched the genome corresponded to Classes 1 to 7 . The SAGEspy program (http://www.osc. edu/research/bioinformatics/projects/sagespy/index.shtml) was used to match the experimental MPSS signatures with the target rice databases to identify the sense, antisense, novel, and alternative transcripts from the MPSS libraries. Clustering analysis was done using in-house programs and Microsoft Access. The bioinformatics pipeline for the SBS data analysis was performed similar to MPSS data analysis with few modifications (Brenner et al. 2000; Meyers et al. 2004a; b; Nobuta et al. 2007). Classification of genes was done using the Kyoto Encyclopedia of Genes and Genomes (KEGG) database (http://www.genome.jp/kegg/).

Identification of antisense, alternative, and novel transcripts

To identify the antisense orientation of the MPSS signatures for the rice reference sequences, we converted all signatures into antisense orientation by a reverse complementation procedure. The antisense signatures from all the MPSS libraries were independently matched against the rice reference sequences. For validating identified antisense signatures from these MPSS libraries, we matched the antisense MPSS signatures against longer antisense rice FLcDNAs available at the KOME database (Osato et al. 2003; http://cdna01.dna.affrc.go.jp/cDNA/Analysis/antisenseweb/ riceantisense.fasta). If a single EST was represented by more than one MPSS signature, then all those signatures were considered as alternative splice/termination of the same gene. The alternative splice forms identified in the MPSS libraries were further confirmed by matching to the rice-alternative-splice-form clusters deposited at http://compbio.dfci.harvard.edu/tgi/cgi-bin/tgi/splnotes.pl? species $=$ Rice.
The reliable signatures that matched the rice genome but did not match rice gene expression databases like KOME FL-cDNAs and TIGR-EST databases were considered to be novel transcripts. Similarly, the genome-matched signatures were considered to be novel genes if they did not match the TIGR ESTs, KOME FL-cDNAs, and TIGR annotated rice genes.

\section{Promoter analysis}

To identify the targets/binding sites of insect-responsive transcription factors and the conserved cis elements among different up-regulated genes, we performed a promoter analysis of the genes commonly induced in both PLA and PLW. Regions $1.0 \mathrm{~kb}$ upstream of the expressed genes were extracted, and the cis elements within these DNA sequences were identified with the "PLACE Signal Scan Search" software (http://www.dna.affrc.go.jp/htdocs/PLACE/, Higo et al. 1999).

Acknowledgments This work was supported by US National Science Foundation awards 0321437 and 0701745 to B.C.M. and G.L.W. and funding from the Arkansas Rice Research and Promotion Board to K.L.K. We thank Dr. Bruce Jaffee for his careful editing of the manuscript.

\section{References}

Arimura G, Tashiro K, Kuhara S, Nishioka T, Ozawa R, Takabayashi J. Gene responses in bean leaves induced by herbivory and by herbivore-induced volatiles. Plant Cell. 2000;19:1096-122.

Arimura G, Kost C, Boland W. Herbivore-induced, indirect plant defenses. Biochim Biophys Acta. 2005;1734:91-111.

Arimura G, Garms S, Maffei M, Bossi S, Schulze B, Leitner M, et al. Herbivore-induced terpenoid emission in Medicago truncatula: concerted action of jasmonate, ethylene and calcium signaling. Planta. 2008;227(2):453-64.

Argandona VH, Chaman M, Cardemil L, Munoz O, Zuniga GE, Corcuera LJ. Ethylene production and peroxidase activity in aphid-infested barley. J Chem Ecol. 2001;27:53-68.

Bede JC, Musser RO, Felton GW, Korth KL. Caterpillar herbivory and salivary enzymes decrease transcript levels of Medicago truncatula genes encoding early enzymes in terpenoid biosynthesis. Plant Mol Biol. 2006;60:519-31.

Brenner S, Johnson M, Bridgham J, Golda G, Lloyd DH, Johnson D, et al. Gene expression analysis by massively parallel signature sequencing (MPSS) on microbead arrays. Nat Biotechnol. 2000;18:630-4.

Broekgaarden C, Poelman EH, Steenhuis G, Voorrips RE, Dicke M, Vosman B. Genotypic variation in genome-wide transcription profiles induced by insect feeding: Brassica oleracea-Pieris rapae interactions. BMC Genomics. 2007;8:239.

Cho SK, Jung KW, Jeung JU, Kang KH, Ship KS, You MK, et al. Analysis of differentially expressed transcripts from plant hopper-infested wild rice (Oryza minuta). Plant Cell Rep. 2005;24:59-67.

de Vos M, Kim JH, Jander G. Biochemistry and molecular biology of Arabidopsis-aphid interactions. Bioessays. 2007;29:871-83. 
De Vos M, Van Oosten VR, Van Poecke RMP. Signal signature and transcriptome changes of Arabidopsis during pathogen and insect attack. Mol Plant Microb Interact. 2005;18:923-7.

Devoto A, Ellis C, Magusin A, Chang HS, Chilcott C. Expression profiling reveals COI1 to be a key regulator of genes involved in wound- and methyl jasmonate-induced secondary metabolism, defense, and hormone interactions. Plant Mol Biol. 2005;58:497513.

Gao LL, Anderson JP, Klingler JP, Nair RM, Edwards OR, Singh KB. Involvement of the octadecanoid pathway in blue green aphid resistance in Medicago truncatula. Mol Plant Microb Interact. 2007;20:82-93.

Gassmann W. Alternative splicing in plant defense. Curr Top Microbiol Immunol. 2008;326:219-33.

Gowda M, Venu RC, Raghupathy MB, Nobuta K, Li H, Stahlberg E, et al. Deep and comparative analysis of the mycelium and appressorium transcriptomes of Magnaporthe grisea using MPSS, RL-SAGE, and oligoarray methods. BMC Genomics. 2006;8 (7):310.

Gowda M, Venu RC, Huameng L, Jantasuriyarat C, Songbiao C, Bellizzi $M$, et al. Magnaporthe grisea infection triggers RNA variation and antisense transcript expression in rice. Plant Physiol. 2007;144:524-33.

Gomez SK, Cox MM, Bede JC, Inoue K, Alborn HT, Tumlinson JH, et al. Lepidopteran herbivory and oral factors induce transcripts encoding novel terpene synthases in Medicago truncatula. Arch Insect Physiol Bioch. 2005;58:114-27.

Halitschke R, Gase K, Hui D, Schmidt DD, Baldwin IT. Molecular interactions between the specialist herbivore Manduca sexta (Lepidoptera, Sphingidae) and its natural host Nicotiana attenuata. VI: microarray analysis reveals that most herbivore- specific transcriptional changes are mediated by fatty acid-amino acid conjugates. Plant Physiol. 2003;131:1894-902.

Harfouche AL, Shivaji R, Stocker R, Williams PW, Luthe DS. Ethylene signaling mediates a maize defense response to insect herbivory. Mol Plant Microb Interact. 2006;19:189-99.

Higo $\mathrm{K}$, Ugawa $\mathrm{Y}$, Iwamoto $\mathrm{M}$, Korenaga $\mathrm{T}$. Plant cis-acting regulatory DNA elements (PLACE) database. Nucleic Acids Res. 1999;27:297-300.

Hilder VA, Gatehouse AMR, Sheerman SE, Barker RF, Boulter D. A novel mechanism of insect resistance engineered into tobacco. Nature. 1987;330:160-3.

Howe GA. Jasmonates as signals in the wound response. J Plant Growth Regul. 2004;23:223-237.

Hudgins JW, Franceschi VR. Methyl jasmonate-induced ethylene production is responsible for conifer phloem defense responses and reprogramming of stem cambial zone for traumatic resin duct formation. Plant Physiol. 2004;135:2134-49.

Jordan T, Schornack S, Lahaye T. Alternative splicing of transcripts encoding toll-like plant resistance proteins - what's the functional relevance to innate immunity? Trends Plant Sci. 2002;7(9):392-8.

Kempema LA, Cui X, Holzer FM, Walling LL. Arabidopsis transcriptome changes in response to phloem-feeding silver leaf whitefly nymphs. Similarities and distinctions in interactions. J Plant Growth Regul. 2007;26:201-9.

Kessler A, Baldwin IT. Plant responses to insect herbivory: the emerging molecular analysis. Annu Rev Plant Biol. 2002;53:299-328.

Korth KL. Profiling the response of plants to herbivorous insects. Genome Biol. 2003;4:221.

Leitner M, Boland W, Mithöfer A. Direct and indirect defences induced by piercing-sucking and chewing herbivores in Medicago truncatula. New Phytol. 2005;167:597-606.

Leon J, Rojo E, Sanchez-Serrano JJ. Wound signaling in plants. J Exp Bot. 2001;52:1-9.

Li Q, Xie Q, Smith-Becker J, Navarre D, Kaloshian I. Mi-1-mediated aphid resistance involves salicylic acid and mitogen-activated protein kinase signaling pathways. Mol Plant Microb Interact. 2006;19:655-664.

Major IT, Constabel CP. Molecular analysis of poplar defense against herbivory: comparison of wound- and insect elicitor-induced gene expression. New Phytol. 2006;172:617-35.

Mello OM, Silva-Filho CM. Plant-insect interactions: an evolutionary arms race between two distinct defense mechanisms. Braz J Plant Physiol. 2002;14:71-81.

Meyers BC, Tej SS, Vu TH, Haudenschild CD, Agrawal V, Edberg $\mathrm{SB}$, et al. The use of MPSS for whole-genome transcriptional analysis in Arabidopsis. Genome Res. 2004a;14:1641-53.

Meyers BC, Vu TH, Tej SS, Matvienko M, Ghazal H, Agrawal V, et al. Analysis of the transcriptional complexity of Arabidopsis by massively parallel signature sequencing. Nat Biotechnol. 2004b; 22:1006-11.

Moran PJ, Cheng Y, Cassell JL, Thompson GA. Gene expression profiling of Arabidopsis thaliana in compatible plant-aphid interactions. Arch Insect Biochem Physiol. 2002;51:182-203.

Nobuta K, Venu RC, Lu C, Belo A, Vemaraju K, Kulkarni K, et al. An expression atlas of rice mRNAs and small RNAs. Nat Biotechnol. 2007;25:473-7.

Osato N, Yamada H, Satoh K, Ooka H, Yamamoto M, Suzuki K, et al. Antisense transcripts with rice full-length cDNAs. Genome Biol. 2003;5:R5.

Pegadaraju V, Knepper C, Reese J, Shah J. Premature leaf senescence modulated by the Arabidopsis PHYTOALEXIN DEFICIENT4 gene is associated with defense against the phloem-feeding green peach aphid. Plant Physiol. 2005;139:1927-34.

Penninckx IAMA, Thomma BPHJ, Buchala A, Metraux JAP, Broekaert WF. Concomitant activation of jasmonate and ethylene response pathways is required for induction of a plant defensin gene in Arabidopsis. Plant Cell. 1998;10:210313.

Ralph S, Oddy C, Cooper D, Yueh H, Jancsik S. Genomics of hybrid poplar (Populus trichocarpa $\times$ deltoides) interacting with forest tent caterpillars (Malacosoma disstria): normalized and fulllength cDNA libraries, expressed sequence tags, and a cDNA microarray for the study of insect-induced defenses in poplar. Mol Ecol. 2006;15:1275-97.

Reymond P, Weber H, Damond M, Farmer EE. Differential gene expression in response to mechanical wounding and insect feeding in Arabidopsis. Plant Cell. 2000;12:707-19.

Reymond P, Bodenhausen N, Van Poecke RM, Krishnamurthy V, Dicke M, Farmer EE. A conserved transcript pattern in response to a specialist and a generalist herbivore. Plant Cell. 2004;16:313247.

Ryan CA. The systemin signaling pathway: differential activation of plant defensive genes. Biochim Biophys Acta. 2000;1477:112-21.

Simon SA, Zhai J, Nandety RJ, McCormick KP, Zeng J, Mejia D, Meyers BC. Short-read sequencing technologies for transcriptional analyses. Annu Rev Plant Biol. 2009;60:305-333.

Stotz HU, Pittendrigh BR, Kroymann J, Weniger K, Fritsche J, Bauke A, et al. Induced plant defense responses against chewing insects. Ethylene signaling reduces resistance of Arabidopsis against Egyptian cotton worm but not diamondback moth. Plant Physiol. 2000;124:1007-17.

Thompson GA, Goggin FL. Transcriptomics and functional genomics of plant defense induction by phloem-feeding insects. J Exp Bot. 2006;57:755-66.

Vega-Sanchez ME, Gowda M, Wang GL. Tag-based approaches for deep transcriptome analysis in plants. Plant Sci. 2007;173:371380 .

Venu RC, Jia Y, Gowda M, Jia MH, Jantasuriyarat C, Stahlberg E, et al. RL-SAGE and microarray analysis of the rice transcriptome after Rhizoctonia solani infection. Mol Genet Genomics. 2007; 278:421-31. 
Voelckel C, Weisser WW, Baldwin IT. An analysis of plant-aphid interactions by different microarray hybridization strategies. Mol Ecol. 2004;13:3187-95.

von Dahl CC, Baldwin IT. Deciphering the role of ethylene in plantherbivore interactions. J Plant Growth Regul. 2007;26:201-9.

Walling LL. The myriad plant responses to herbivores. J Plant Growth Regul. 2000;19:195-216.

Winz RA, Baldwin IT. Molecular interactions between the specialist herbivore Manduca sexta (Lepidoptera, Sphingidae) and its natural host Nicotiana attenuata: insect-induced ethylene reduces jasmonate-induced nicotine accumulation by regulating putrescine N-methyltransferase transcripts. Plant Physiol. 2001;125:2189-202.
Yuan JS, Köllner TG, Wiggins G, Grant J, Degenhardt J, Chen F. Molecular and genomic basis of volatile-mediated indirect defense against insects in rice. Plant J. 2008;55(3):491-503.

Zarate SI, Kempema LA, Walling LL. Silverleaf whitefly induces salicylic acid defenses and suppresses effectual jasmonic acid defenses. Plant Physiol. 2007;143:866-75.

Zhang F, Zhu L, He G. Differential gene expression in response to brown planthopper feeding in rice. J Plant Physiol. 2004;161:5362.

Zheng SZ, Dicke M. Ecological genomics of plant-insect interactions: from gene to community. Plant Physiol. 2008; $146(3): 812-7$. 